\title{
Light-weight spherical mirrors for Cherenkov detectors
}

\author{
E. Cisbani ${ }^{\mathrm{a}}$, S. Colilli ${ }^{\mathrm{a}}$, R. Crateri ${ }^{\mathrm{a}}$, F. Cusanno ${ }^{\mathrm{a}}$, R. Fratoni ${ }^{\mathrm{a}}$, S. Frullani ${ }^{\mathrm{a}}$,
}

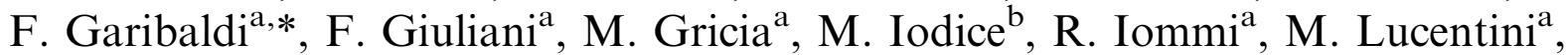
A. Mostarda ${ }^{\text {a }}$, L. Pierangeli ${ }^{\mathrm{a}}$, F. Santavenere ${ }^{\mathrm{a}}$, G.M. Urciuoli ${ }^{\text {a }}$, R. De Leo ${ }^{\mathrm{c}}$,

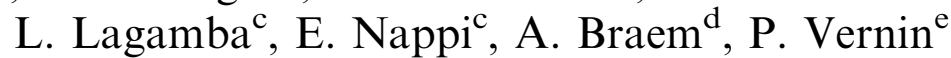

${ }^{a}$ Physics Laboratory, Instituto Superiore di Sanità and Sezione INFN Sanità, viale Regina Elena 299, I-00161 Rome, Italy

${ }^{\mathrm{b}}$ INFN Sezione di Roma III, via Della Vasca Navale 84, I-00147 Rome, Italy

${ }^{\mathrm{c}}$ Dipartimento Interateneo di Fisica e Sezione INFN Bari, via Amendola 173, I-70126 Bari, Italy

${ }^{\mathrm{d}}$ CERN, Geneva, Switzerland

${ }^{\mathrm{e}}$ CEA, Gif sur Yvette, France

Received 10 May 2002; received in revised form 26 August 2002; accepted 3 September 2002

\begin{abstract}
Light-weight spherical mirrors have been appositely designed and built for the gas threshold Cherenkov detectors of the two Hall A spectrometers. The mirrors are made of a $1 \mathrm{~mm}$ thick aluminized plexiglass sheet, reinforced by a rigid backing consisting of a phenolic honeycomb sandwiched between two carbon fiber mats epoxy glued. The produced mirrors have a thickness equivalent to $0.55 \%$ of radiation length, and an optical slope error of about $5.5 \mathrm{mrad}$. These characteristics make these mirrors suitable for the implementation in Cherenkov threshold detectors. Ways to improve the mirror features are also discussed in view of their possible employment in RICH detectors.
\end{abstract}

(C) 2002 Elsevier Science B.V. All rights reserved.

Keywords: Mirrors; Cherenkov detectors

\section{Introduction}

In the design of Cherenkov counters the mirrors play a relevant role because they must collect the produced Cherenkov light with minimum loss.

In the past many techniques have been employed to build spherical mirrors for Cherenkov detectors, using as substrate either a glass or a plastic material.

\footnotetext{
*Corresponding author. Fax: + 39-06-49387075.

E-mail address: franco.garibaldi@iss.infn.it (F. Garibaldi).
}

Although glass allows to achieve superior surface optical qualities [1], mirrors built with "lighter" materials are often mandatory when the Cherenkov detectors are followed by other downstream detectors.

In these cases, the mirrors are generally manufactured exploiting low density materials, such as acrylics, which are unexpensive and relatively easy to machine. However they easily experience deformations, thus entailing unpredictability of the optical performance when large surfaces are needed. Consequently, in order to avoid optical 
distortions, it is mandatory to use a sort of rigid support, made of either foams, or composite structures, such as sandwiches of fiberglass mats and honeycomb [2-4].

In this paper we describe a novel technique exploited to build very light-weight spherical mirrors $\left(380 \times 480 \mathrm{~mm}^{2}\right.$ rectangularly shaped, with a curvature radius of $R=90 \mathrm{~cm}$ ) for the gas threshold Cherenkov detectors of the two TJNAF Hall A spectrometers. Such detectors have been designed with the aim to separate electrons from pions up a momentum of $4 \mathrm{GeV} / c$, with high efficiency and high rejection ratio [5-7]. Their details, characteristics and performances have been described elsewhere [6].

\section{Principles of construction}

The procedure often employed for manufacturing light-weight mirrors is based on the use of two moulds, one negative for providing the spherical shape to the plexiglass, the other positive to achieve the required stiffness by glueing the carbon fiberglass and the phenolic honeycomb sandwich [3].

The major difficulty raised by this technique is an imperfect matching between the negative and the positive moulds, i.e. between the honeycombcarbon fiber structure and the plexiglass. Hence, an alternative and less expensive procedure has been implemented and described in this paper. The plexiglass sheet is shaped to a spherical segment with the desired radius of curvature $(90 \mathrm{~cm})$ by means of the process of "heating and vacuum forming". Subsequently the curved plexiglass sheet is placed on a positive aluminum mould where various carbon fiberglass mats and the phenolic honeycomb are epoxy glued on its back. The final structure is then achieved by employing the "vacuum bagging technique" which exploits the air pressure for the adhesive curing.

These new forming and curing techniques have been shown to minimize the problems caused by the different "shrinking" properties of the employed materials (plexiglass, honeycomb, carbon fibers). In Fig. 1 a typical mirror built with this new technique is shown.

\subsection{Plexiglass forming}

Although a standard heating and vacuum technique was used to slump the plexiglass sheets to the desired shape, particular care was necessary to achieve satisfactory results.

A special device (Fig. 2) was appositely realized for heating and vacuum forming the plexiglass sheets: an oven with reflecting walls, made in such a way as to provide an uniform distribution of the temperature on the plexiglass sheet, which is fundamental for attaining the correct spherical shape.

The features of such a device were driven by the plexiglass solidification temperature of $150^{\circ} \mathrm{C}$. Initially the plexiglass sheet is heated to $190^{\circ} \mathrm{C}$, close to the maximum of the thermoplastic phase temperature $\left(200^{\circ} \mathrm{C}\right)$, then the plexiglass sheet is moved to the box with a circular hole in the lower part of the device where the desired radius of curvature is achieved by vacuum pumping. The process has to be as fast as possible in order to avoid the cooling to a temperature close to $150^{\circ} \mathrm{C}$. Hence, the box is located as close as possible to the oven in order to be able to start the vacuum formation just some seconds after reaching the right temperature. The temperature is continuously monitored in many points of the oven. The desiderated radius of curvature is obtained by using a laser beam positioned at a well defined distance from the plexiglass sheet; the vacuum pumping is stopped when the laser light starts to graze the plexiglass sheet (Fig. 2). Bringing the plexiglass at room temperature, its curvature radius may decrease. Further pumpings and laser checks are necessary in order to obtain the desiderated final curvature radius.

The precision on the curvature radius obtained with this method is around $1 \%$ for a $1 \mathrm{~mm}$ thick plexiglass sheet. A higher precision is not necessary in the present application since the distance between photomultipliers (PMT) and mirrors can be easily adjusted.

\subsection{Composite structure forming}

The final composite structure was obtained by applying the "vacuum bagging" technique on a 


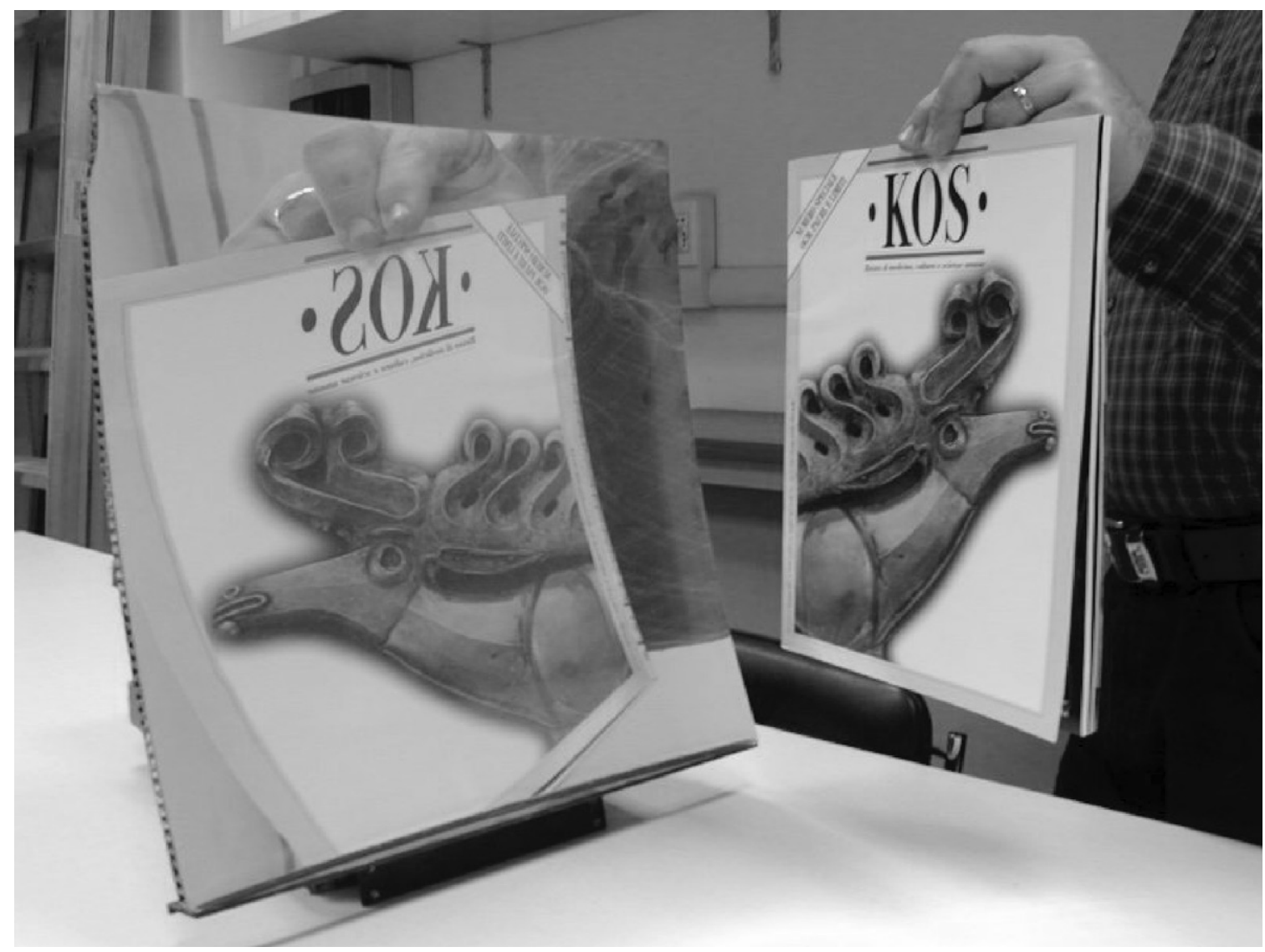

Fig. 1. A typical mirror.

mould consisting of a spherical section of aluminum, machined and polished up to a roughness of $0.8 \mu \mathrm{m}$ (see Fig. 3).

To avoid any "telegraphing" effect (roughness transfer from the mould to the plexiglass surface) and to correct for casual small variations in the radius of curvature, the mould had to be optically polished. A chromium layer $(0.02 \mathrm{~mm}$ thick) was applied on the mould before this treatment. The result is shown in Fig. 3.

The procedure adopted is described in the following.

First a good matching between the plexiglass sheet and the mould curvatures is checked: the plexiglass sheet is put on the mould; if the curvature radius of the plexiglass sheet is smaller than the mould curvature radius (contact at the edge, missing contact at the center) the plexiglass sheet is thrown away; if the curvature radius of the plexiglass sheet is not smaller than the mould curvature radius (contact on the center, possible missing contact on the edge) the plexiglass sheet is thrown away in case of edge distance bigger than $1 \mathrm{~mm}$. The mismatch occurs only in few cases thus proving the suitability of the technique.

Three layers of epoxy glue/carbon fiber mats were used for the first sheet of the sandwich. Particular care had to be used in "mixing" the carbon fiber mat and epoxy glue to avoid any wrinkling that could generate deformations likely to be transferred to the plexiglass sheet. Later the honeycomb sheet is added, followed by three more sheets of epoxy glue/carbon fiber mats. 


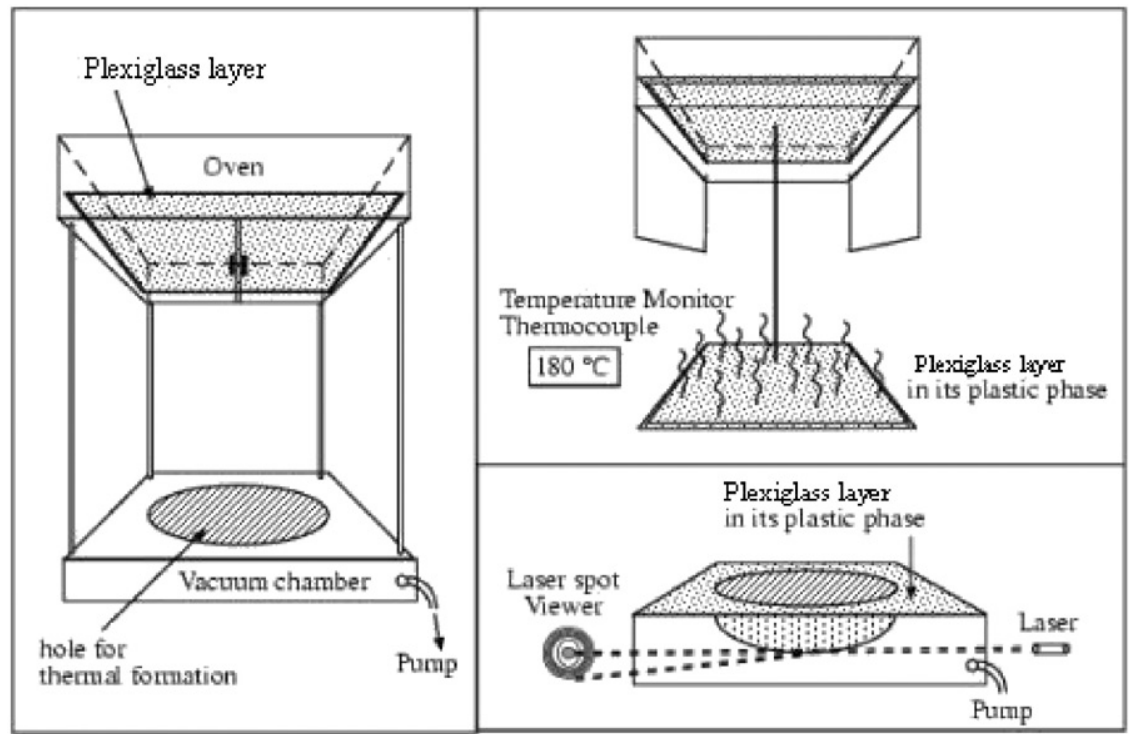

Fig. 2. The device used for the plexiglass forming. The three phases of the manufacturing procedure are shown.

The whole setup (mould-plexiglass-sandwich) is then wrapped in a vacuum bag and brought to an underpressure of $300 \mathrm{~mm} \mathrm{Hg}$. A higher value would result in an excess of telegraphing, a lower one would bring to a faulty shaping. In this way the optical quality of the mirror was optimized.

Heating, sometimes used to accelerate the polymerization of the epoxy glue, was not employed in this procedure because any temperature increase showed a deterioration of the optical mirror quality, probably caused by a "differential" shrinking coming from the polymerization process.

Phenolic honeycomb sheets of the "flex-core" type by Hexcel $^{1}$ were used. Such a honeycomb configuration is the most suitable in our application because it can be shaped in tridimensional compound curvatures with minimum shrinking effects due to its particular cell shape. The cell density is 50 cells per inch squared. The thickness chosen $(13 \mathrm{~mm})$ is the best compromise between the stiffness optimization and the material thickness minimization.

\footnotetext{
${ }^{1}$ Hexcel Corporation.
}

Carbon mats were used because of their better shaping properties with respect to the conventional webs. The fibers were randomly placed, not woven, so that it was possible to achieve a good mixing with the glue, avoiding, to a certain extent, some wrinkling that could deteriorate the optical properties of the mirrors. Moreover such units are very light, while being rather rigid.

The thickness of the plexiglass sheets $(1 \mathrm{~mm})$ was a compromise between the wanted stiffness and the necessity to have mirrors as "light" as possible with good optical quality. Thinner $(0.5 \mathrm{~mm})$ plexiglass sheets, although commercially available, show a not guaranteed surface quality. On the other hand, $2 \mathrm{~mm}$ plexiglass sheets show a better surface quality and minimization of the "telegraphing" effect, although they give rise to higher deformations caused by the difficulty in forming/shaping structures with a higher rigidity.

In spite of all the described cares, residual shrinking was still present in the final mirrors, however not to an extent compromising their optical performances. The final result is a composite structure of $230 \mathrm{mg} / \mathrm{cm}^{2}$, equivalent to $0.55 \%$ of a radiation length, which, to our knowledge, is the smallest value recorded in the literature. 


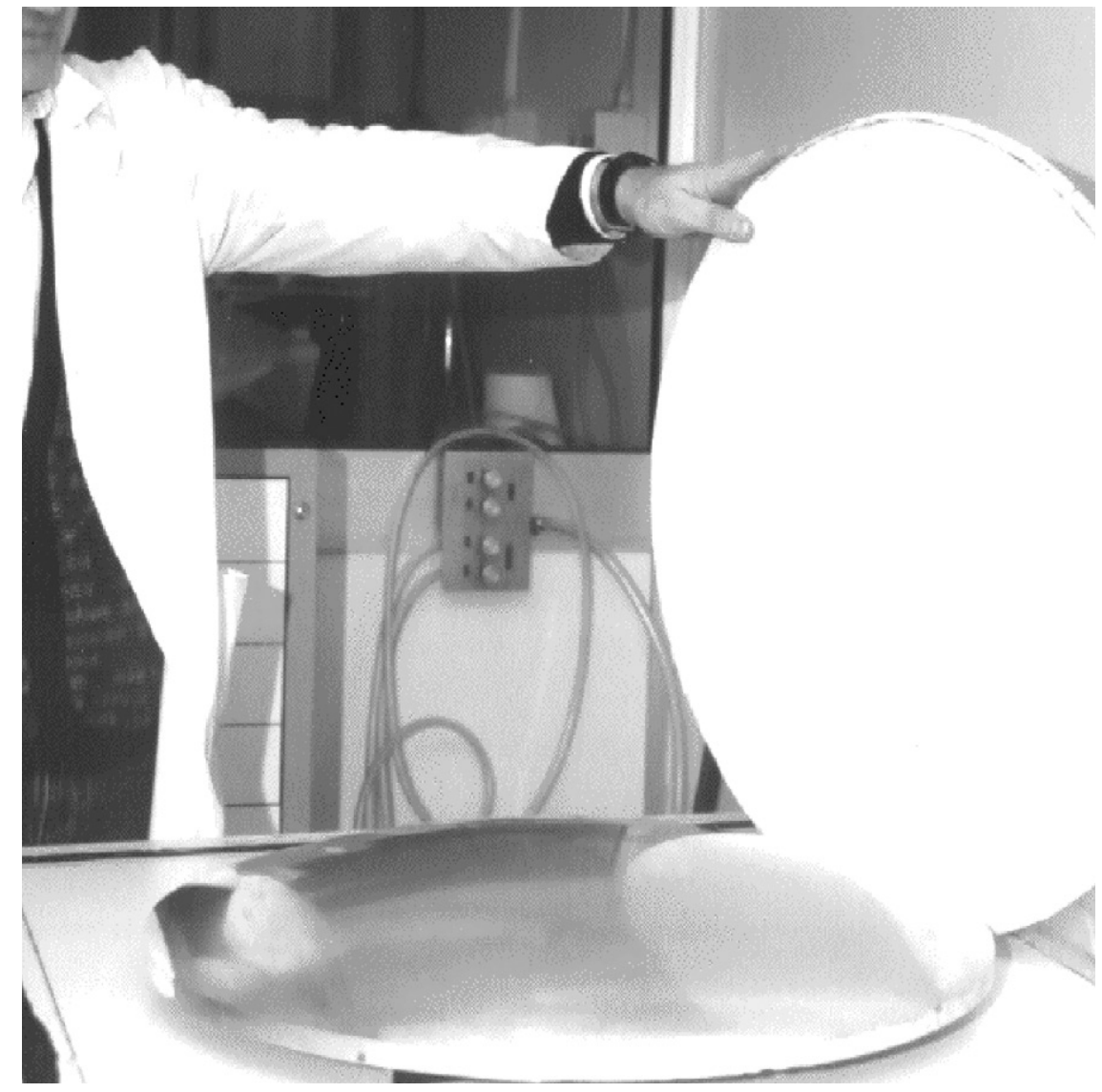

Fig. 3. The mould.

\subsection{Aluminization}

The aluminization process was performed at CERN (at the TA1 Thin Film Laboratory) by evaporating $70 \mathrm{~nm}$ of $\mathrm{Al}$ followed by a coating of evaporated $\mathrm{MgF}_{2}, 30 \mathrm{~nm}$ thick.

Aluminum was chosen as main reflecting material because of its well known properties in the wavelength interval of interest $(220-600 \mathrm{~nm})$ (curve with crosses in Fig. 4(a)). By varying the $\mathrm{MgF}_{2}$ thickness it is possible to shift the reflectivity peak as a function of wavelength (see other curves in Fig. 4(a)). The optimal peak position (or $\mathrm{MgF}_{2}$ thickness) is the one that makes the reflectivity peak to overlap the maximum of the PMT quantum efficiency (reported in Fig. 4(b)). The transparency of the used radiator $\left(1 \mathrm{~m}\right.$ of $\mathrm{CO}_{2}$ gas), reported with full triangles in Fig. 4(a), shows a low $\lambda$-cut, and thus it does not influence the reflectivity.

Some preliminary measurements were made to test the influence of the support material on the final mirror reflectivity. To this aim three different supports, namely a glass sheet, a plexiglass sheet, and a mylar foil were aluminized as described above ( $70 \mathrm{~nm}$ of $\mathrm{Al}+30 \mathrm{~nm}$ of $\mathrm{MgF}_{2}$ ) and their reflectivity measured. The results are summarized in Fig. 5. It is evident that glass is the best support, although the plexiglass sheet is close to it, and superior to the mylar foil.

The reflectivity measurements were made at CERN in a dedicated apparatus (Fig. 6) containing a UV light source, a monochromator, $\mathrm{a} \mathrm{CaF}_{2}$ focussing lens (automatically positioned in the 


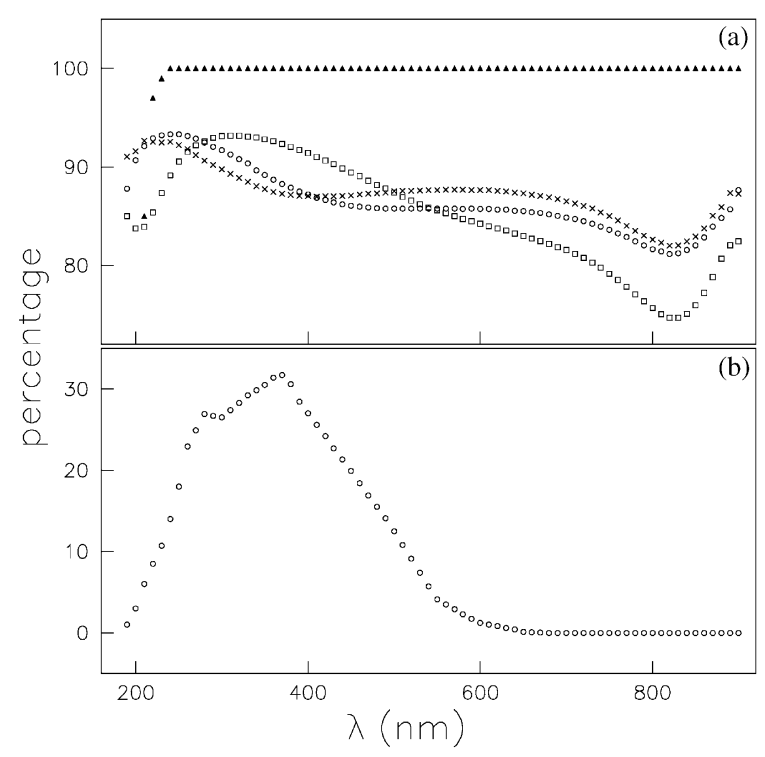

Fig. 4. (a) Reflectivity of a plexiglass mirror with an evaporation of: $70 \mathrm{~nm} \mathrm{Al}$ (crosses), $70 \mathrm{~nm} \mathrm{Al}$ and $10 \mathrm{~nm} \mathrm{MgF}$ (empty circles), $70 \mathrm{~nm} \mathrm{Al}$ and $30 \mathrm{~nm} \mathrm{MgF}_{2}$ (empty squares), together with the transparency of a $1 \mathrm{~m}$ long $\mathrm{CO}_{2}$ gas (full triangles). (b) The quantum efficiency of a BURLE PMT with a bialkali photocathode.

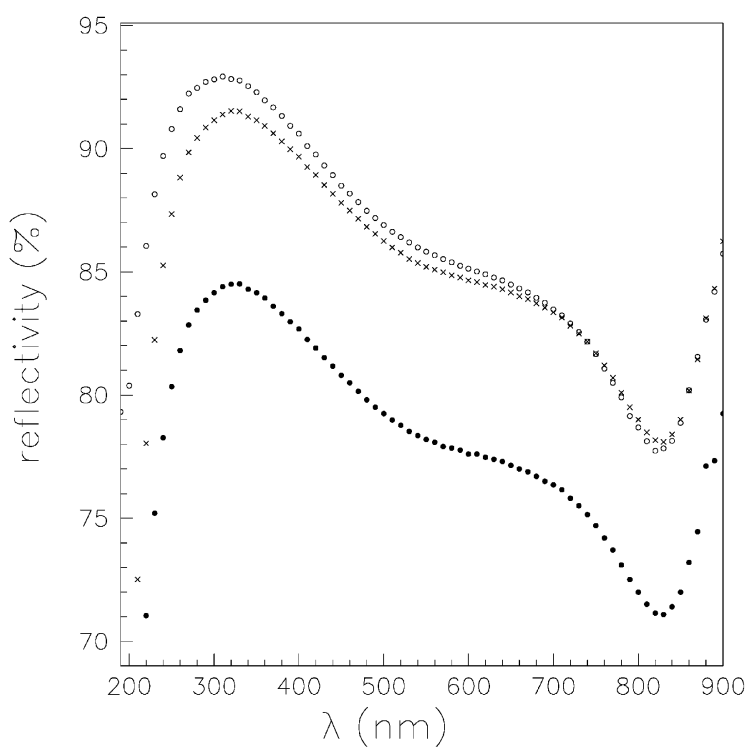

Fig. 5. Reflectivity as a function of the photon wavelength for mirrors aluminized with $70 \mathrm{~nm} \mathrm{Al}$ and $30 \mathrm{~nm} \mathrm{MgF}_{2}$ and produced with different substrates: glass (empty circles), plexiglass (crosses), mylar (full circles). Mylar substrates show a stronger light diffusion caused by their surface roughness. focus of the selected wavelength), a rotating mirror and a diaphragm, two photomultipliers (one for source intensity monitoring, one for measuring the reflectivity), and a mechanically adjustable sample holder. After mounting the mirror onto the vacuum vessel, the sample holder is rotated until the reflected light hits the center of the PMT. This is ensured by maximizing the PMT counting rate, which is depending on its front aperture.

\section{Optical tests}

Two types of tests have been performed to check the mirror optical qualities.

\subsection{Integral techniques}

The first technique exploits a point like source positioned in the center of the spherical mirror in such a way as to illuminate the whole mirror. The image is registered with a CCD camera located in the sphere center, just close to the source. Any deviation from a point like image, both for size and shape, is caused by macro-deformations of the mirror surface sphericity. The slope error is given by the ratio between the radius size of the image spot and the mirror radius $(91 \mathrm{~cm})$.

Some of the recorded image spots are shown in Fig. 7. The spot size is always less than $10 \mathrm{~mm}$ in diameter, resulting in a slope error lower than 5.5 mrad.

This technique, useful for surveying the macroscopic defects, does not reveal diffusion losses in the reflection caused by microscopic irregularities of the mirror surface. We have surveyed these diffusion losses in a second "integral" technique obtained by illuminating the whole mirror surface with a parallel beam and registering the image in the mirror focus. The parallel beam was provided by using an auxiliary mirror $(560 \mathrm{~mm}$ in diameter and $2673 \mathrm{~mm}$ in focal length) with a point source $(0.4 \mathrm{~mm}$ in diameter) in its focus, as shown in Fig. 8. If a CCD is placed in the focus, spots similar and half in size of those in Fig. 7 are achieved, thus confirming slope errors measured by the previous technique. The diffusion losses are obtained by replacing the CCD in Fig. 8 with a 


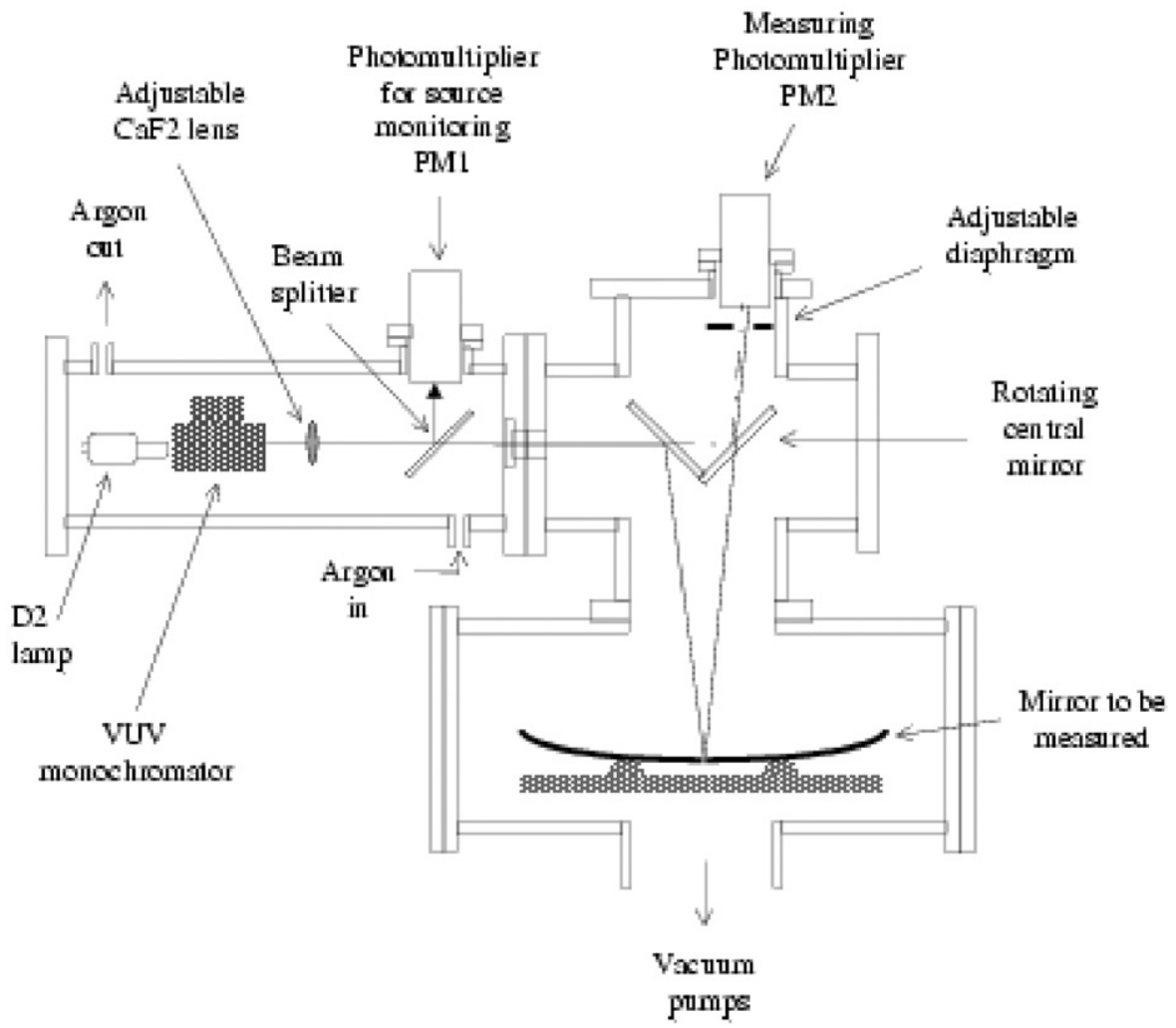

Fig. 6. Schematics of the apparatus used for measuring the mirror reflectivity.

$8 \mathrm{~mm}$ photocell seen through an adjustable diaphragm, and by measuring the fraction of the incident parallel light reflected in the circular corona with radius from 4.5 to $8 \mathrm{~mm}$, i.e. outside the main spot ( $9 \mathrm{~mm}$ in diameter). The criterion of considering the mirror as having acceptably small microscopic imperfections when the reflected fraction in the circular corona is less than $10 \%$ was adopted. This technique has been used to select 300 mirrors for the DELPHI RICH detector [1]. The entire set of 20 mirrors produced with our technique passed this second integral test.

This second technique allows to obtain also the "actual" focus of the mirrors, defined as the one where the spot size is at its minimum. The resulting radius of curvature of the produced mirrors is $91 \mathrm{~cm}$, very close to the "nominal" one.

The two integral technique measurements showed that the optical quality of the mirrors is sufficiently good for their use in (focusing) threshold Cherenkov counters.

\subsection{Tests on the detector box}

The mirrors were mounted into the detector boxes and an "on site" optical test was performed. A light ray was produced along the generatrices of a cone, close from a geometrical point of view to the Cherenkov light produced by the particle traversing the Hall A spectrometers. The shape of the light spot was recorded at the PMT position (Fig. 9a) and compared with the one generated by a Monte Carlo calculation (Fig. 9b). The comparison of the shapes of the two figures is very good, suggesting that all the light emitted is collected by the mirrors on the PMT. 

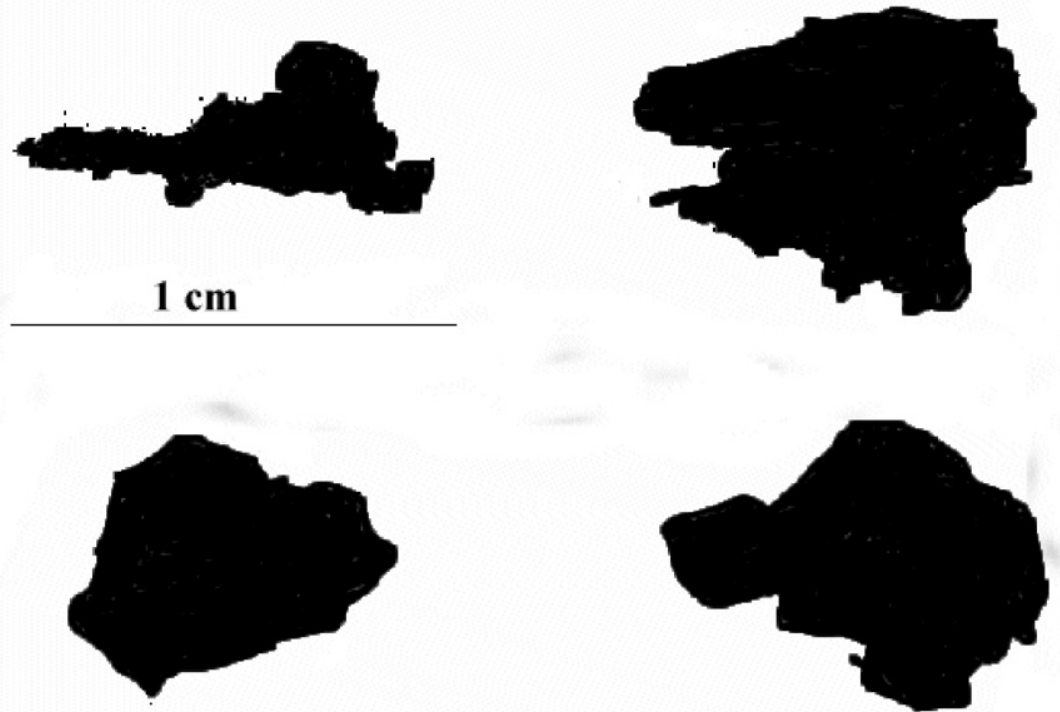

Fig. 7. Typical spots from different mirrors.

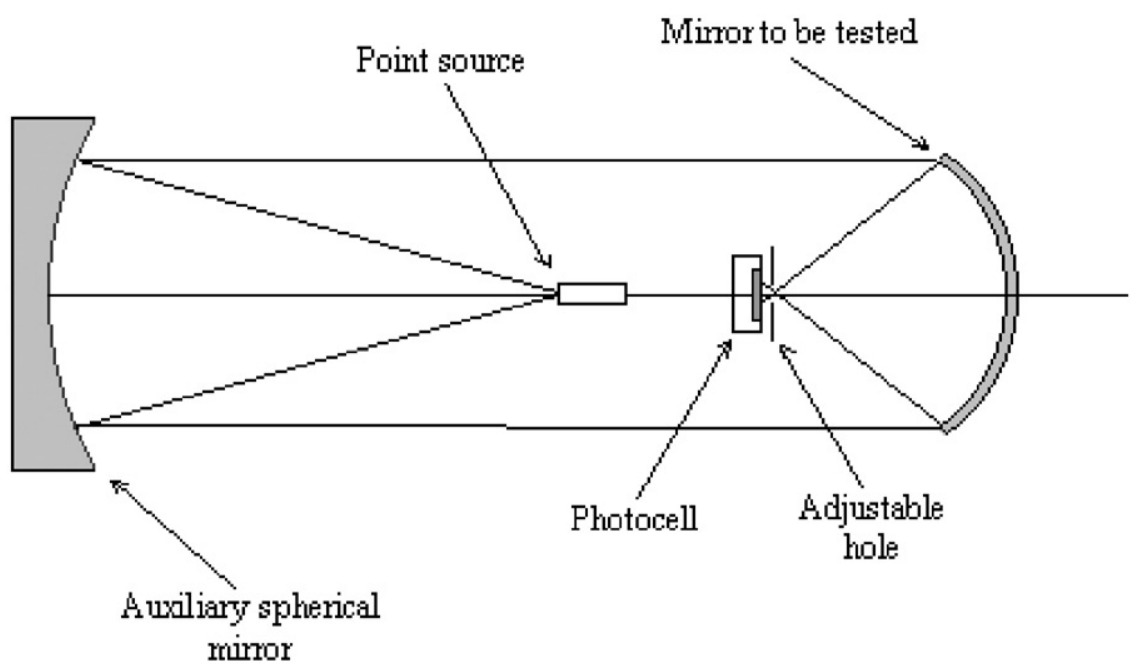

Fig. 8. Device used for measuring the spot size.

\section{Improvements}

A $R \& D$ program is in progress with the aim to improve the described technique for realizing RICH detector mirrors for kaon physics [8] by reducing the slope error, thus matching the required RICH performances [7].

In fact it seems that in principle almost all the effects causing the enlargement and the deformation of the spot shape may be eliminated or 
(a)
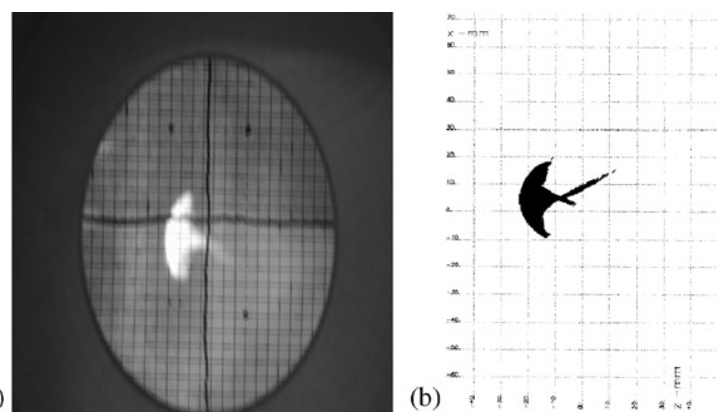

Fig. 9. Optical test in the detector box. The actual spot (a) and its Monte Carlo simulation (b).

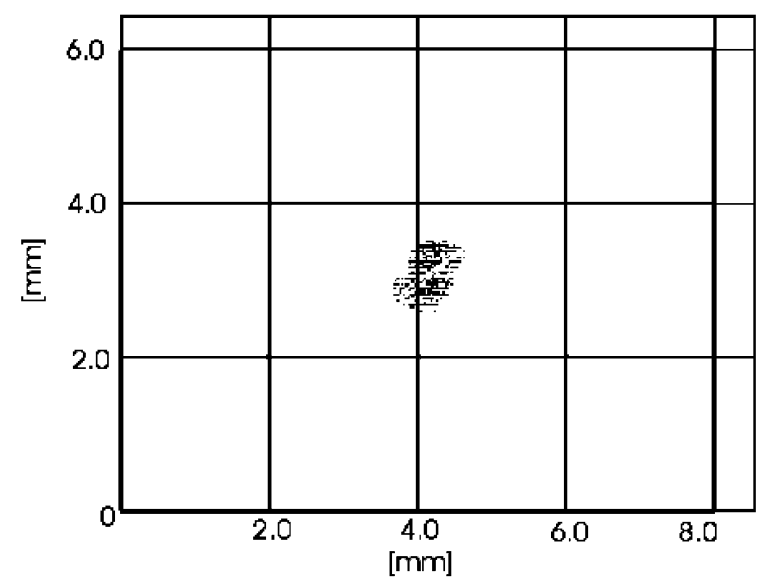

Fig. 10. Spot measured with a mirror of improved quality.

minimized. Most of the limitations are due to the quality of the plexiglass sheet surfaces which is not as good as that of glass. Moreover some degree of telegraphing effect will be present anyway, due to the softness of the plexiglass.

It has been proved that some of the problems decrease for mirrors with both smaller dimensions $\left(20 \times 20 \mathrm{~cm}^{2}\right.$ for example) and bigger curvature radii $(R=180 \mathrm{~cm})$. Spot images with diameter smaller than $2 \mathrm{~mm}$ have been obtained for mirrors of such dimensions (an example is reported in Fig. 10), corresponding to a slope error of $0.55 \mathrm{mrad}$, comparable with that of glass mirrors for RICH detectors [1]. A mosaic of small mirrors could be used to cover the wanted reflecting surfaces. Other effects causing the enlargement of the spot and its deviation from the circular shape are the shrinking caused by the epoxy glue, and the residual dust in the working environment.

The curvature radii of our mirrors has a limited precision. We are trying to solve this problem by using a computer to control the plexiglass shaping and to improve the reproducibility of our forming procedure.

\section{Conclusions}

Very light-weight mirrors have been built for threshold Cherenkov detectors. A relatively simple and unexpensive technique, completely realized in a non-specialized small laboratory, has been used.

The mirrors are made by $1 \mathrm{~mm}$ thick spherical $(R=900 \mathrm{~mm})$ plexiglass sheets $\left(380 \times 480 \mathrm{~mm}^{2}\right)$ with a rigid backing made by a sandwich with phenolic honeycomb and carbon fiber mat/epoxy glue. The main characteristics of the produced mirrors are: $0.55 \%$ of radiation length and an optical slope error of about $5.5 \mathrm{mrad}$. Although these are good features for applications to Cherenkov threshold detectors, some improvements of the technique are necessary and have been discussed in order to use these mirrors in focusing RICH detectors which require mirrors of higher optical qualities.

\section{Acknowledgements}

We are particularly grateful to A. Liesenfeld and M. Meoni for interesting discussions and suggestions during the various phases of the mirror construction.

\section{References}

[1] P. Baillon, et al., Nucl. Instr. and Meth. A 276 (1989) 492.

[2] R. Fohrman, et al., Nucl. Instr. and Meth. 184 (1981) 319.

[3] A. Liesenfeld, personal communication.

[4] A. Braem, M.E. Kostrikov, Radiation Transparent Mirrors for RICH, IHEP 93-129.

[5] Conceptual Design Report, Southern University Research Association (SURA), April 1990. 
[6] M. Iodice, et al., Nucl. Instr. and Meth. A 411 (1998) 223.

[7] F. Garibaldi, et al., Hadron identification at Jefferson Lab, in: C. Williams, T. Ypsilantis (Eds.), Proceedings of the 36th Workshop of the INFN Eloisatron Project, Erice (TP), Italy.
[8] S. Frullani, F. Garibaldi, J.J. LeRose, P. Markowitz, T. Saito, High Resolution 1p Shell Hypernuclear Spectroscopy, Experimental CEBAF Proposal PR-94-107, 1994. 